\title{
Simulation strategies for the LHC ATLAS experiment
}

\author{
Andy Buckley \\ University of Edinburgh \\ for the ATLAS Collaboration
}

IEEE Nuclear Science Symposium, Knoxville, 2010-11-02
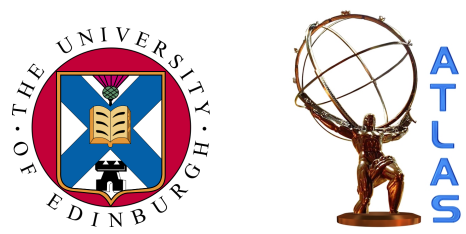


\section{The ATLAS detector}

General purpose detector designed to cover all the expected physics channels at LHC using $\mathrm{p}-\mathrm{p}$ collisions with energies up to $14 \mathrm{TeV}$ and $\mathrm{Pb}-\mathrm{Pb}$ collisions at $5.5 \mathrm{TeV} /$ nucleon.

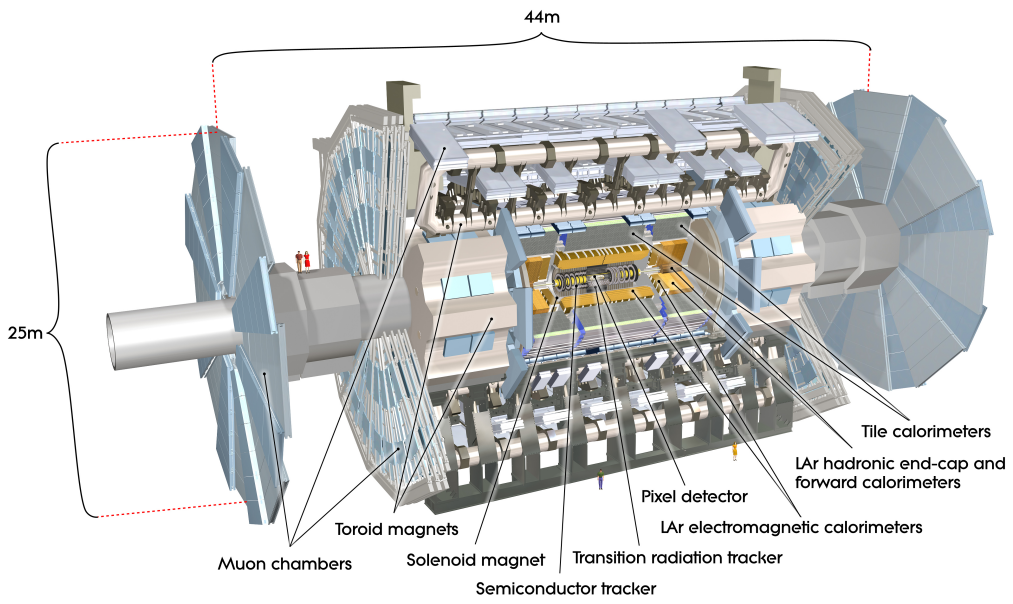




\section{ATLAS operation}

- Experiment performing according to design specifications.

- Excellent operational efficiency.

- Extremely good understanding of the apparatus and detector simulation for this stage of operation.

\section{ATLAS Detector Status}

\begin{tabular}{l|c|c|}
\hline Subdetector & Number of Channels & Approximate Operational Fraction \\
\hline Pixels & $80 \mathrm{M}$ & $97.3 \%$ \\
\hline SCT Silicon Strips & $6.3 \mathrm{M}$ & $99.2 \%$ \\
\hline TRT Transition Radiation Tracker & $350 \mathrm{k}$ & $97.1 \%$ \\
LAr EM Calorimeter & $170 \mathrm{k}$ & $98.1 \%$ \\
Tile calorimeter & 9800 & $96.9 \%$ \\
Hadronic endcap LAr calorimeter & 5600 & $99.9 \%$ \\
Forward LAr calorimeter & 3500 & $100 \%$ \\
\hline LVL1 Calo trigger & 7160 & $99.9 \%$ \\
\hline LVL1 Muon RPC trigger & $370 \mathrm{k}$ & $99.5 \%$ \\
\hline LVL1 Muon TGC trigger & $320 \mathrm{k}$ & $100 \%$ \\
\hline MDT Muon Drift Tubes & $350 \mathrm{k}$ & $99.7 \%$ \\
CSC Cathode Strip Chambers & $31 \mathrm{k}$ & $98.5 \%$ \\
\hline RPC Barrel Muon Chambers & $370 \mathrm{k}$ & $97.0 \%$ \\
\hline TGC Endcap Muon Chambers & $320 \mathrm{k}$ & $98.6 \%$ \\
\hline
\end{tabular}

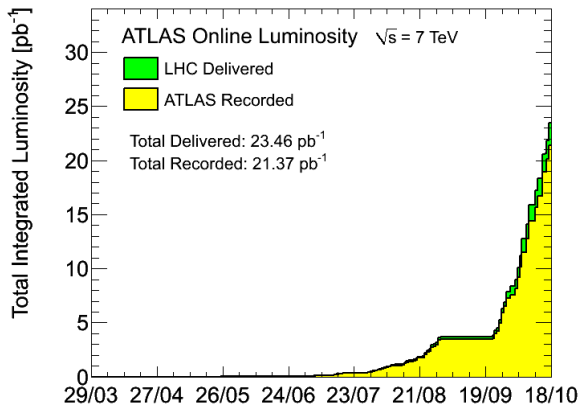

Day in 2010 


\section{Events}

Highest-mass QCD dijet at ATLAS: $3.1 \mathrm{TeV} / \mathrm{c}^{2}$ for two leading jets (red and green)!

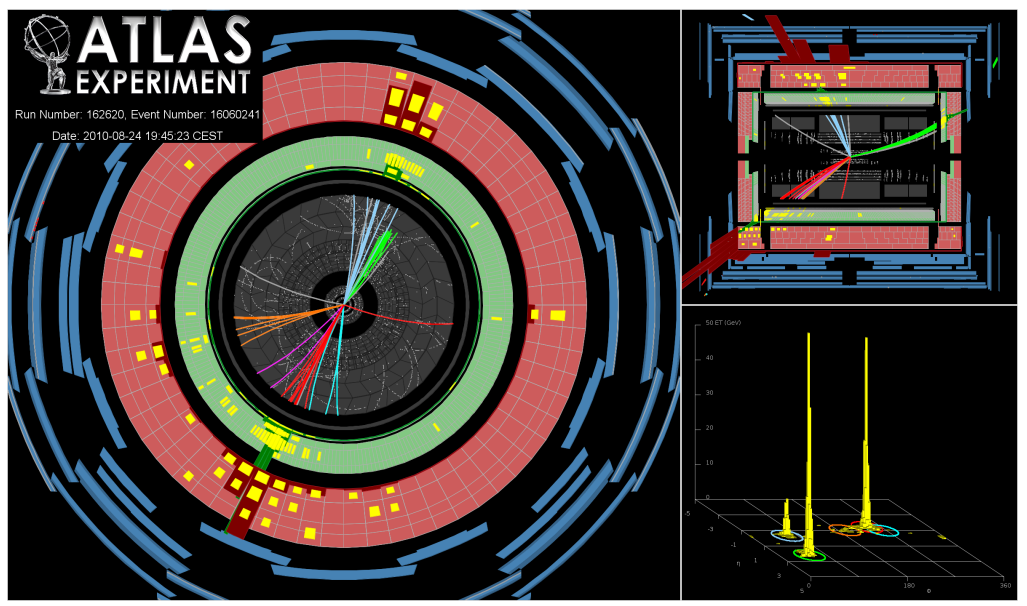




\section{Events}

$\mathrm{Z} \rightarrow \mu^{+} \mu^{-}$in $7 \mathrm{TeV}$ LHC collisions at ATLAS

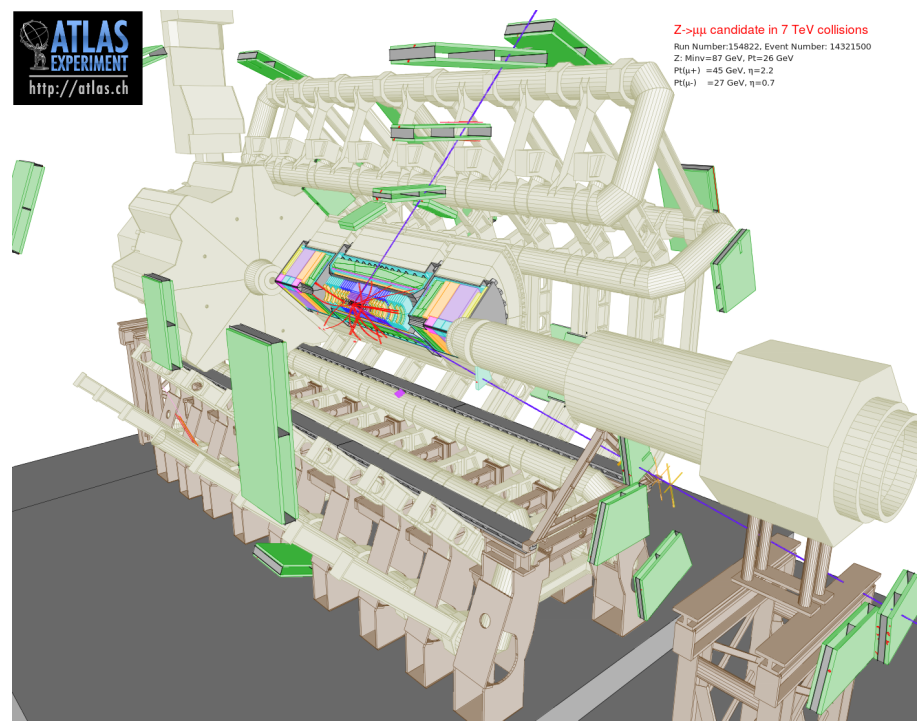




\section{ATLAS simulation framework}

- Simulation of physics events / detector via common ATLAS software framework (Athena)

- Detector simulation based on Geant4

- Multiple layouts supported: ATLAS +

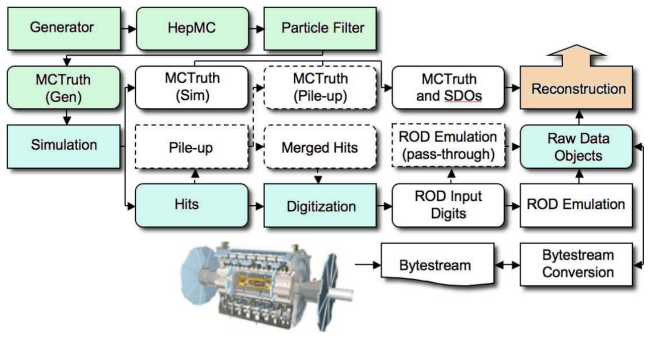
test beam setups, etc.

ATLAS GeoModel: shared geometry translation to G4/reco

Framework used for large scale ( $\sim 500 \mathrm{M}$ event) production on LHC Computing Grid: $\sim 8 \mathrm{M}$ event/day. Failure rate $<10^{-6}$. 


\section{Some alternative layouts for ATLAS simulation}
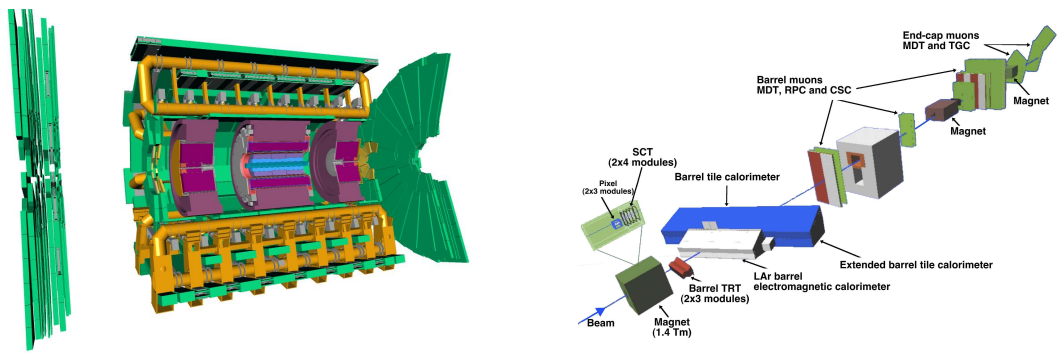

Cosmics commissioning

Combined test-beam 


\section{ATLAS simulation details}

- Event generation interfaces for $\sim 40$ generator codes: maintained, validated, and tuned

- Truth-level HepMC events smeared with beam-spot parameters and tracked through detector description by Geant4 application $\Rightarrow$ HITS

- Detector description parameters and conditions data from tagged database entries. Including variation tags for test stands, ATLAS commissioning, extra material, misalignments, etc.

- Special cases: cosmics, forward detectors, beam halo and cavern background (require extra particle transport); $R$-hadrons, stopped gluinos, Q-balls, monopoles...

- Pile-up, halo and non-beam backgrounds included at digitization stage: merging of HITS files - now with bunch structure. 


\section{ATLAS core simulation framework}

- Full sim currently using G4 9.2.patch02.atlas04 - 12 patches incorporated for issues reported by/affecting ATLAS. Next major update: straight to G4 9.4.

- Several access layers: G4 classes augmented with Athena features via FADS - Framework for ATLAS Detector Simulation - and configured/steered by an Athena algorithm / Python scripting layer.

- Particle scoring done in Athena det-specific classes. Enables optimisation for task, e.g. LAr calorimeter groups G4 HITS before persistency to save memory.

- Currently re-writing aspects of these layers: initial requirement was for interactive usage at a prompt. In practice never used this way - developers $\rightarrow$ users in ATLAS, and most usage now via fixed steering "transform" scripts. 


\section{Fast simulation}

G4 sim very CPU/memory intensive: some analyses require higher stats than possible $\Rightarrow$ fast sim

- ATLFAST-I: direct HepMC $\rightarrow$ reco smearing by parameterised detector $/$ reco resolution

- ATLFAST-II: parameterised particle showers (FastCaloSim) and simplified tracking (FATRAS)

- Fast G4: use "frozen" pre-simulated shower libraries for $10 \mathrm{MeV}-1 \mathrm{GeV}$

- Can also simulate e.g. only inner detector for track-only

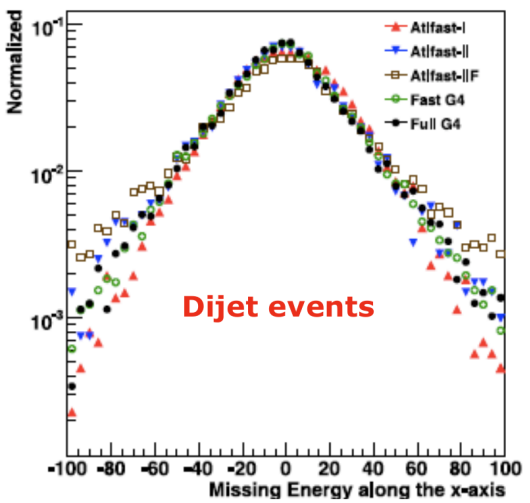
analyses 


\section{Fast simulation CPU performance}

Relative timings of $\mathrm{t} \overline{\mathrm{t}}$ ATLAS detector sim events:

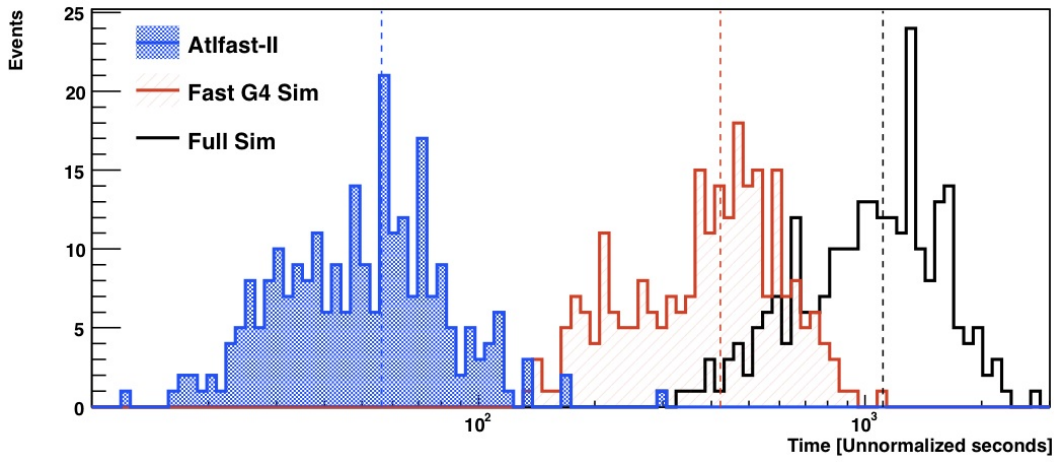




\section{Simulation comparison to data}

Excellent understanding of detector:

Pixel detector:

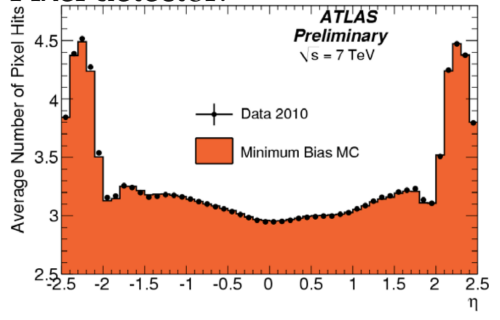

Silicon central tracker (SCT):

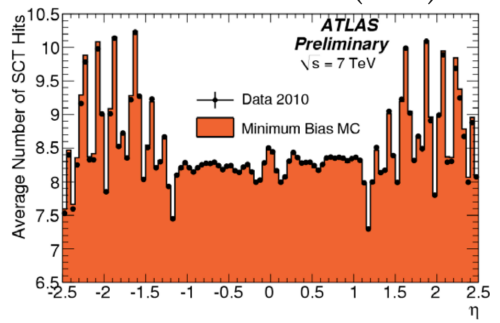




\section{Simulation comparison to data}

Excellent understanding of detector:

Close-up of secondaries in 1st pixel layer (data, sim):
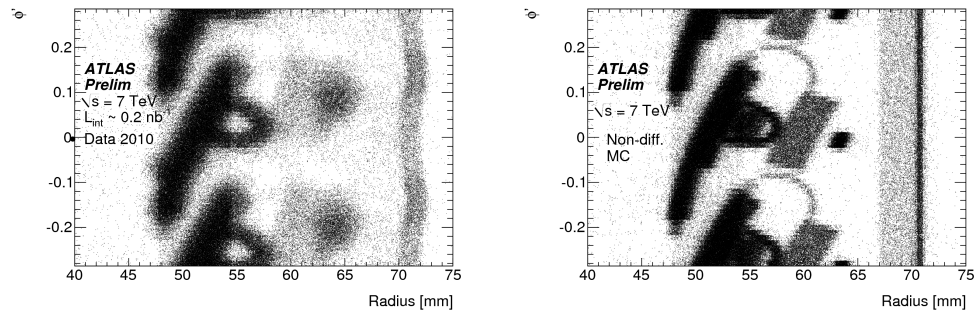


\section{Simulation tuning to data}

Geometry fixes from design to correct sim photon conversion radius:

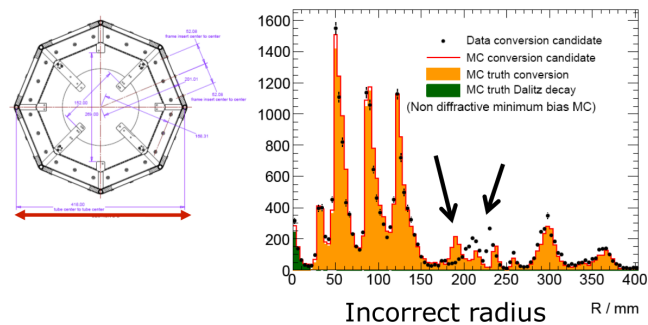

Also two fixes for anomalous G4 events with energy non-conservation:

- Jets at 55 and $67 \mathrm{GeV}$ found in $\mathrm{J0}\left(p_{\perp} \in[7--14] \mathrm{GeV}\right)$ slice! No truth match: energy not conserved in $\mathrm{G} 4 \gamma+\mathrm{Pb} \rightarrow \pi^{+} \pi^{-}+\mathrm{X}$

- In $\pi^{+}+\mathrm{He} \rightarrow \mathrm{pp}+X$, protons with $47 \mathrm{TeV}$ each! Pretty clearly a bug!

- G4 produced patches: in production and in new G4 releases. 


\section{Performance monitoring and optimisation}

- Performance monitoring with a variety of physics and particle-gun events

- Done for all new releases, G4 builds, etc.; robustified statistics

- Sim CPU used to be production bottleneck, now reco disk space
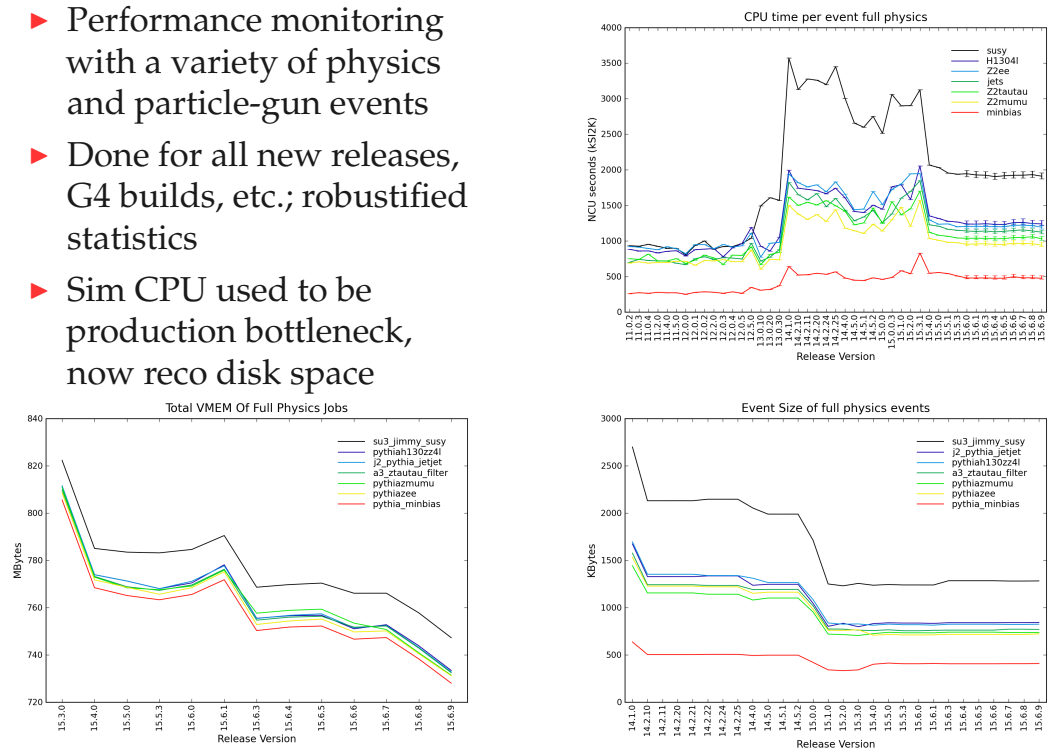


\section{CPU focus: field stepper system}

- RK4 adaptive steppers used for particle propagation. Calls to $B$ field map $\Rightarrow \sim 20 \%$ of sim CPU

- 11 calls in ClassicalRK4 stepper $\Rightarrow 4$ using AtlasRK4 stepper with analytic error calc

- AtlasRK4 also has field-caching in a fixed radius: further field-call reduction

- Further reduction to only 2 calls possible with G4 NystromRK4 stepper

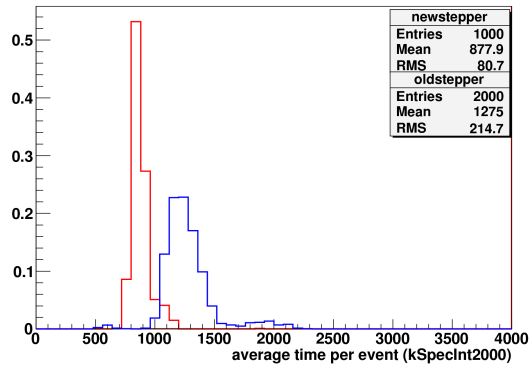

- Also "stepper dispatcher": choose stepper based on region, track PID, energy, proposed length 


\section{Summary}

- Simulation framework very successful: excellent detector description, improving performance: simulating $1 \mathrm{M}$ events per day in ATLAS MC production.

- Work is never done! Optimisation, new G4 versions, new physics list validation, fast simulation, structural re-working.

- Current development effort: forward detectors, cavern bg, stepper dispatcher, pile-up bunch structure and data-MC overlays. ATLAS upgrade.

- Described in full detail in ATLAS sim paper: arXiv:1005.4568 